\title{
Cluster: A New Application for Spatial Analysis of Pixelated Data for Epiphytotics
}

\author{
Scot C. Nelson, ${ }^{\dagger}$ Iulian Corcoja, and Sarah J. Pethybridge
}

First author: College of Tropical Agriculture and Human Resources, Department of Tropical Plant and Soil Sciences, University of Hawaii at Manoa, Honolulu, HI 96822; second author: AQUASoft Inc., Bucharest, Romania; third author: Cornell University, School of Integrative Plant Science, Section of Plant Pathology \& Plant-Microbe Biology, Cornell University, Geneva, NY 14456.

Accepted for publication 7 August 2017.

\begin{abstract}
Spatial analysis of epiphytotics is essential to develop and test hypotheses about pathogen ecology, disease dynamics, and to optimize plant disease management strategies. Data collection for spatial analysis requires substantial investment in time to depict patterns in various frames and hierarchies. We developed a new approach for spatial analysis of pixelated data in digital imagery and incorporated the method in a stand-alone desktop application called Cluster. The user isolates target entities (clusters) by designating up to 24 pixel colors as nontargets and moves a threshold slider to visualize the targets. The app calculates the percent area occupied by targeted pixels, identifies the centroids of targeted clusters, and computes the relative compass angle of orientation for each cluster. Users can deselect anomalous clusters manually and/or automatically by specifying a size threshold value to exclude smaller targets from the analysis. Up to 1,000 stochastic simulations randomly place the centroids of each cluster in ranked order of size (largest to smallest) within each matrix while preserving their calculated angles of orientation for

the long axes. A two-tailed probability $t$ test compares the mean intercluster distances for the observed versus the values derived from randomly simulated maps. This is the basis for statistical testing of the null hypothesis that the clusters are randomly distributed within the frame of interest. These frames can assume any shape, from natural (e.g., leaf) to arbitrary (e.g., a rectangular or polygonal field). Cluster summarizes normalized attributes of clusters, including pixel number, axis length, axis width, compass orientation, and the length/width ratio, available to the user as a downloadable spreadsheet. Each simulated map may be saved as an image and inspected. Provided examples demonstrate the utility of Cluster to analyze patterns at various spatial scales in plant pathology and ecology and highlight the limitations, trade-offs, and considerations for the sensitivities of variables and the biological interpretations of results. The Cluster app is available as a free download for Apple computers at iTunes, with a link to a user guide website.
\end{abstract}

Quantification of spatial attributes and patterns in plant pathology and scientific disciplines such as biology, ecology, and geography is essential for hypothesis testing. In plant pathology, a comprehensive understanding of how spatial patterns evolve forms the basis of effective disease management strategies that minimize crop loss (Hughes 1987; Madden et al. 2007; McRoberts et al. 2003). The types of data used to quantify epidemics usually dictate the techniques and approaches applied to spatial analysis (Baddeley and Turner 2005; Madden et al. 2007; Pielou 1977; Ripley 1981; Schwanck and Del Ponte 2016). The common goal unifying these techniques is to provide robust, statistical evidence that at a point in time the spatial pattern of disease depicts specific, scale-dependent spatial heterogeneity (Ferrandino 1998; Turechek and McRoberts 2013).

Depending upon the types of data collected (binary versus continuous) to quantify an epidemic, the statistical approaches generally fall into two broad categories that analyze the spatial pattern in relation to the scale of the sampling (Turechek and McRoberts 2013). One approach attempts to convert an overall pattern into a statistic that is not scalar and based on fit of the data to a theoretical distribution (Hughes and Madden 1992). This approach applies where binary data (e.g., disease incidence) indicate the frequency distribution of the proportion of diseased individuals within a sampling unit (Hughes and Madden 1993; Madden and Hughes 1994, 1995, 1999; Turechek and Mahaffee 2004; Turechek et al. 2011). Implementation of these approaches provides a direct measure of heterogeneity of disease incidence at the sampling unit level (Hughes and Madden 1992; Shah et al. 2017a; Turechek and Madden 2003).

†Corresponding author: S. C. Nelson; E-mail: snelson@hawaii.edu

(C) 2017 The American Phytopathological Society
Correlation or 'geostatistics-based' analyses provide information to complement distributional techniques at the sampling unit level or above and can indicate the degree or strength of aggregation for a nonrandom pattern (Turechek and Madden 1999a, b). Examples of correlation-based analyses commonly used in plant pathology include runs (e.g., ordinary and median) analyses (Gibbons and Chakraborti 2010; Madden et al. 1982; Swed and Eisenhart 1943), interpolation algorithms such as semivariograms (Isaaks and Srivastava 1989), Morisita's index (Morisita 1959), the variance-to-mean ratio (GreigSmith 1952), two-dimensional distance class analysis (Gray et al. 1986; Nelson et al. 1992), spatial correlation analysis (Ferrandino 1996, 1998; Gottwald et al. 1992), and spatial analysis by distance indices (Li et al. 2012; Perry 1995, 1998; Perry et al. 1999). Collectively, results of these analyses are highly dependent upon the predefined spatial hierarchy (Gosme and Lucas 2009a, b; Hughes 1987; Hughes et al. 1997; Kriss et al. 2012; Shah et al. 2017b; Turechek and McRoberts 2013; Willocquet and Savary 2004). Nelson (1996) also described a correlation-based technique to provide information on the directional components of spatial pattern. Many attempts to represent and quantify the spatial patterns of plant disease epidemics therefore include assumptions regarding the binary classification of a plant or sampling unit. However, using disease incidence or severity to represent a spatial pattern in an artificially defined, two-dimensional space such as a linear transect or grid of given dimensions may oversimplify or misrepresent the broader-scale spatial attributes of an epidemic and the projected crop loss.

Recent advances in image analysis and processing have enhanced our understanding of infection processes and disease development, including the ability to use color to represent and quantify disease severity (Barbedo 2013, 2014; Bock et al. 2010; Lamari 2002; Mutka and Bart 2015; Pethybridge and Nelson 2015). Imaging science also provides for transformational approaches to characterizing 
plant-pathogen interactions (Kuska et al. 2015) and the detection and differentiation of plant diseases and abiotic stress on a common host, facilitating high-throughput phenotyping (Leucker et al. 2016; Mahlein et al. 2010, 2012). Similarly, the digital agriculture revolution has delivered complementary hardware to capture highdensity, spatial resolution imagery on various platforms (Wolfert et al. 2017). Routine and readily accessible platforms for capturing aerial images include unmanned aerial systems, low elevation fixed wing aircraft or from satellites. These platforms allow the interrogation of images and pixelated data to enhance understanding of biological processes and plant disease epidemiology. For example, Schwanck and Del Ponte (2016) used digital image analysis to measure lesion attributes and analyze spatial patterns by illustrating the approach on leaves through multiple steps that transfer data between platforms. In that study, attributes of discrete lesions were extracted following manipulation of individual photographs within an image analysis program, Assess (Lamari 2002). Data were interrogated through standard statistical software such as the SPATSTAT package of R (Baddeley and Turner 2005) and standalone programs (Perry 1995; Perry et al. 1999). The benefits of adopting and encapsulating this analytical, conceptual framework within a single platform were further highlighted by Schwanck and Del Ponte (2016).

The objective of our study was to extend the image analysis algorithms implemented within the Leaf Doctor app for calculation of percent disease severity (Pethybridge and Nelson 2015) to spatial analysis by developing a stand-alone quantitative assessment tool for analysis of pixel-based data derived from imagery. A complementary objective of this study was to demonstrate the utility and trade-offs of this approach for applications in plant pathology across different sampling unit scales and the potential extension of this technique into affiliated disciplines of ecology and geography.

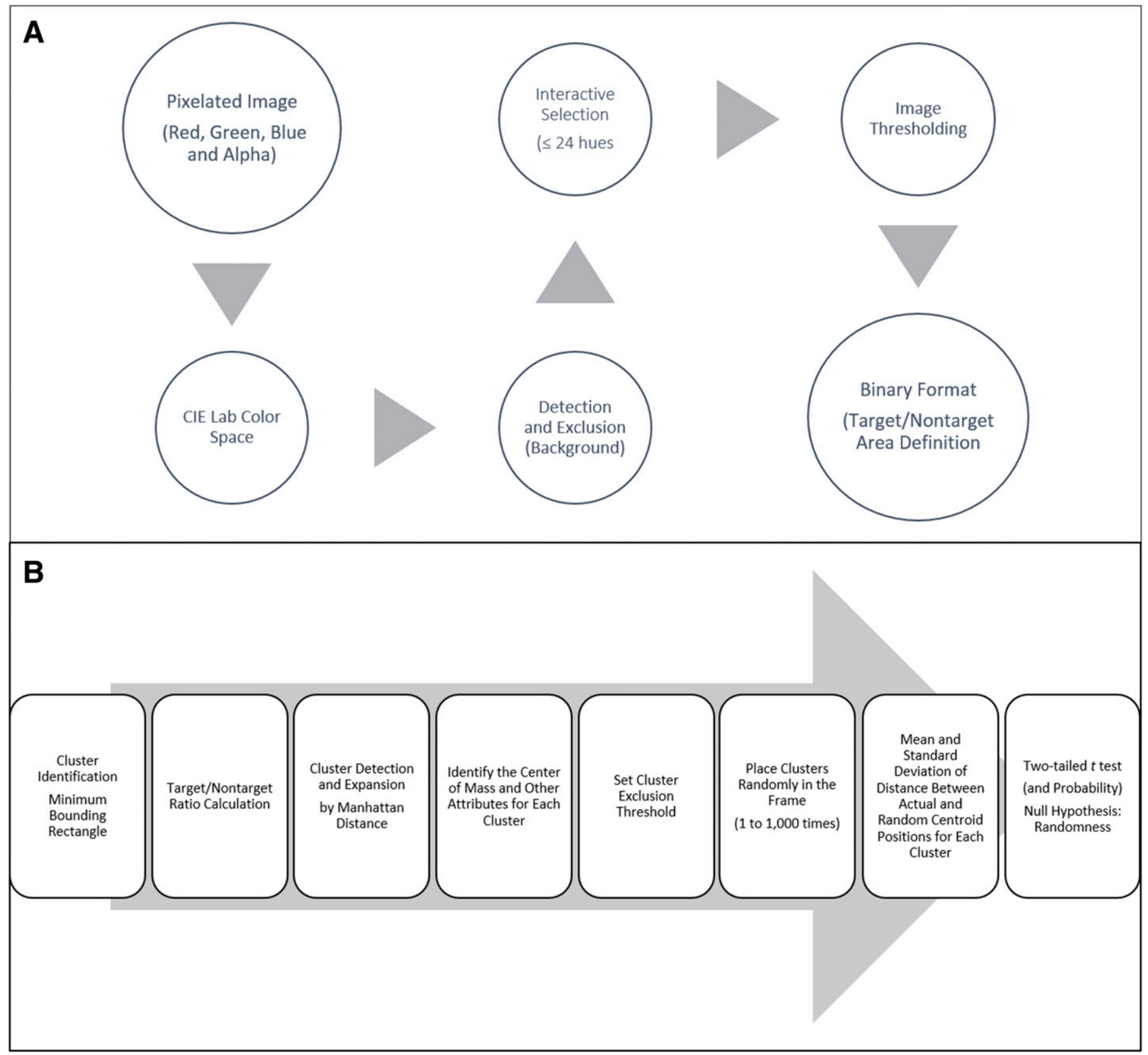

Fig. 1. Workflows used by the Cluster app involved in A, processing a digital photograph supplied in original color space format (red, green, blue and alpha channels) to binary format; and $\mathbf{B}$, computations made from the binary format to definition of the cluster attributes and executing an analysis to test a null hypothesis of a random spatial pattern of the clusters using a two-tailed $t$ test and associated probability value. 


\section{MATERIALS AND METHODS}

Development of the Cluster app. The Cluster app tests a null hypothesis of a random spatial pattern of clusters (target areas defined by pixels from digital imagery within a frame of interest) and summarizes the attributes of clusters to facilitate additional hypothesis testing. The principal app coding languages were Swift $2.3(80 \%)$ and Objective-C and $\mathrm{C} / \mathrm{C}++(20 \%)$, refactored and compiled with the latest SDK for macOS 12.12 and Xcode 8.0. Objective- $\mathrm{C}$ and $\mathrm{C} / \mathrm{C}++$ languages handled low-level computations. A summary of the workflow for the Cluster app is shown in Figure 1.

Image processing. Data from a digital image loads into the computer's main access and graphical processing unit (GPU) for conversion from original color space format (red, green, blue, and alpha [RGBA]) to the nonlinearly compressed CIE lab color space ( $L \times a \times b$; where $L=$ lightness; and $a$ and $b=$ color-opponent dimensions [Hunter 1948a, b]). This enables color balance corrections by modifying output curves in the $a$ and $b$ components, or adjusting the lightness contrast of the $L$ component (Jain 1989). The alpha channel in the image preserves and stores information for each pixel. Image processing enables detection and exclusion of the background color from the spatial analysis by applying algorithms to the converted Lab image. The background color detection algorithm has four steps executed in the following order: (i) an extractable $3 \times 3$ matrix of pixels derives from each of four corners of an image; (ii) an average color computes from every matrix, resulting in four different colors; (iii) the four colors compare with each other with a specified threshold; and (iv) the algorithm determines if the four average colors for each corner are similar, close or equal to each other using Euclidean distance (Gower 1982, 1985). If the four average colors are similar or equal to each other, an additional average is computed to represent the background of the image. If the color difference exceeds an established threshold, the algorithm ignores any further stages of image background color detection and removal. If the background detection algorithm identifies a color, a flood-filling algorithm is implemented in all directions across the image using a queue as the data structure for storing the pixels for analysis (Treuenfels 1994). Initiation of the algorithm begins at the four corners of the image and results in equilibration of the final as the background color. The flood-fill algorithm determines the area connected to a given node in a multidimensional array. The algorithm identifies adjacent pixels in the image matrix that are connected to the starting point. The image's alpha channel identifies every pixel that is close or equal to the background color as 0 to 200 and 255 for the object color. The app ignores the color of the selected background pixels when the user selects a color within the image to compute the selected/unselected color ratio or the count of unselected colors. When the app renders an image for preview, it draws background color-marked pixels to match the window's color. The user can preview and interrogate the image by (i) selection of one or multiple colors (healthy tissues); (ii) removal of selected colors; and (iii) adjustment of the threshold between selected and unselected colors using a slider. A zoom and pan function assists in image interrogation.

Color selection. The user interactively identifies pixels as target/ nontarget (i.e., diseased/healthy) areas. The selected colors are displayed sequentially below the image as hued circles above the threshold slider. The user may select up to 24 colors to represent nontarget hues. Color selection transforms the image into a binary format facilitating definition of a border between the target and nontarget areas. This process is analogous to image thresholding (Sezgin and Sankur 2004). In this procedure, the app replaces pixels within an image with a black pixel if the color intensity $I_{i, j}$ is less than a fixed constant $T$ (that is $I_{i, j}<T$ ), or white if the pixel color intensity is greater than the fixed constant (Sezgin and Sankur 2004). Each user-selected color is loaded into the GPU memory and passed as an argument for computation of target and nontarget areas (i.e., diseased and healthy tissues). This step depends on the
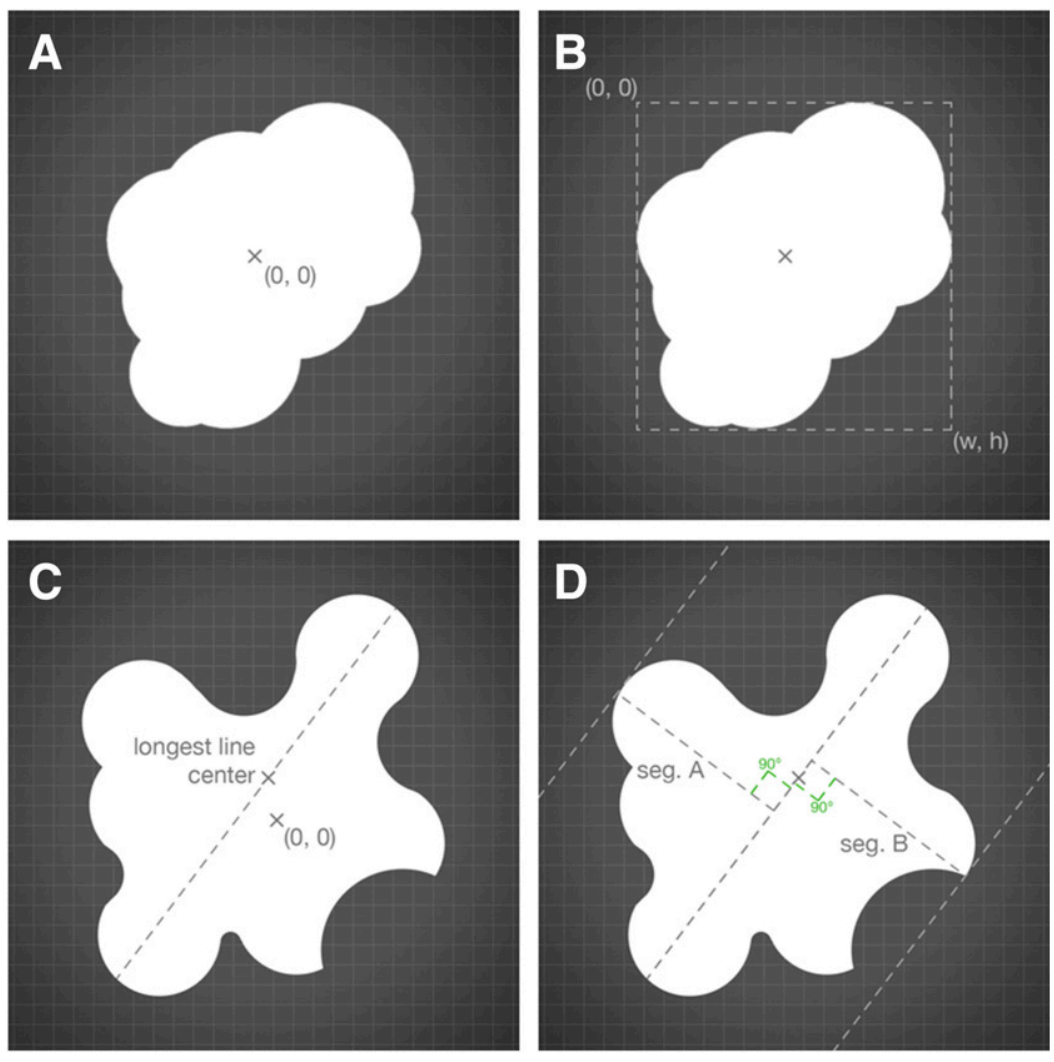

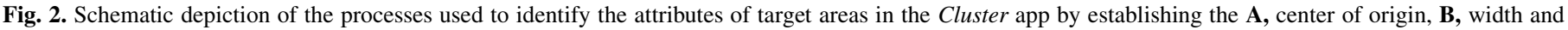
height, $\mathbf{C}$, longest center line, and $\mathbf{D}$, individual dimensions. 
following criteria: (i) color computation occurs only for the lab image, (ii) the app ignores background pixels as it compares each target pixel to the selected color, and (iii) if a pixel is close or similar to at least one user-selected color it is considered a nontarget area. The app conducts color comparisons using Euclidean distance through implementation of the $\Delta E$ CIE76 formula (Sharma 2003). This equation relates to a known set of CIE $L \times a \times b$ coordinates. Therefore, preserving $\left(L_{1} \times a_{1} \times b_{1}\right)$ and $\left(L_{2} \times a_{2} \times b_{2}\right)$, the Cluster app establishes the threshold using

$$
\Delta \mathrm{E}_{a b}=\sqrt{\left(L_{2}-L_{1}\right)^{2}}+\left(a_{2}-a_{1}\right)^{2}+\left(b_{2}-b_{1}\right)^{2}
$$

If $\Delta \mathrm{E}_{a b}<\varepsilon$, where $\varepsilon=$ actual threshold, the app selects the color. The app does not select the color if the difference is larger or equal to the threshold $\left(\Delta \mathrm{E}_{a b} \geq \varepsilon\right)$. The threshold used for this equation in Cluster is $\Delta \mathrm{E} \times a b=0.0255$, approximating to a just noticeable difference of 2.3 (Sharma 2003).

Threshold adjustment. The user adjusts the color threshold value by dragging the threshold slider between 0 and $100 \%$, where $0 \%$ corresponds to no adjustment of color threshold (no area selected) and $100 \%$ indicates selection of the entire area. If the threshold has a value of $0(\Delta \mathrm{E} \times a b<0)$, the compared colors differ. If the threshold has a value of $255(100 \%)$, the compared colors do not differ. Selected threshold values load into the GPU and pass as an argument for computation of target and nontarget areas.

Image processing. When the selected colors accurately fill and represent the target areas (i.e., the clusters) with blue, the image loads from the GPU to the random-access memory of the central processing unit. The output image is in RGBA format with the alpha channel representing processed data. The values 0 (background color), 128 (target areas colors), and 255 (nontarget area colors) store and record every pixel in the alpha channel.

Computation of pixel types. Pixel type computation by the app within the target and nontarget areas initiates by establishing a minimum-bounding rectangle (MBR) (O'Rourke 1985). In brief, this algorithm stores the raw data of the image in a single array of pixels represented in two-dimensions according to width and height. To increase processing speed, the image parses by multiple threads with every thread responsible for a specific allotment of data. The image splits into multiple parts of equal size by dividing raw data by $2 x$ the number of computer cores. These data parse by summing every pixel type representing the background, target and nontarget areas, and computing the top-left and bottom-right pixel position of the target/nontarget pixel type within each thread. The MBR for the image in the absence of background color has an origin $\left(x_{\min }, y_{\min }\right)$ and dimensions (length $=x_{\max }-x_{\min }$; width $=y_{\max }-$ $\left.y_{\text {min }}\right)$. The app calculates final position and size of the MBR by evaluating the minimum and maximum position of the pixels, where $t l=$ pixel at top-left position; $b r=$ pixel at bottom-right; and $n=$ number of threads using

$$
\begin{aligned}
& x_{\text {min }}=\operatorname{MIN}\left(x_{1}^{t l}, x_{2}^{t l}, x_{3}^{t l}, \ldots x_{n}^{t l}\right) ; y_{\text {min }}=\operatorname{MIN}\left(y_{1}^{t l}, y_{2}^{t l}, y_{3}^{t l}, \ldots y_{n}^{t l}\right) \\
& x_{\text {max }}=\operatorname{MAX}\left(x_{1}^{b r}, x_{2}^{b r}, x_{3}^{b r}, \ldots x_{n}^{b r}\right) ; y_{\text {max }}=\operatorname{MAX}\left(y_{1}^{b r}, y_{2}^{b r}, y_{3}^{b r}, \ldots . y_{n}^{b r}\right)
\end{aligned}
$$

Calculation of target/nontarget ratio. The ratio of target to nontarget areas calculates by dividing the number of pixels associated with each area and is reported as a percentage:

$$
r=\frac{p x_{\text {diseased }}}{p x_{\text {healthy }}} \times 100
$$

The accuracy of the algorithm for pixel selection and calculation of percent disease was found to be analogous to other standard software used in phytopathometry (Pethybridge and Nelson 2015).
Cluster detection and analysis. The app defines a cluster as a set of adjacent pixels connected according to the principles of Manhattan distance (Garg and Sharapov 2002). In brief, Manhattan distance states that connected pixels may be in a horizontal, vertical, or diagonal plane within a $3 \times 3$ neighborhood matrix. The app therefore represents a cluster object by an array, preserving the positions of each pixel from the original image with the following properties: (i) the quantified spatial position is relative to the image origin $(0,0)$, (ii) bounded by a MBR or cluster frame, (iii) a center of mass, (iv) an orientation angle, (v) length represented by the longest axis, (vi) width represented by the length of the longest axis perpendicular to the longest axis, (vii) defined edge points, and (viii) is an array of pixel points.

Detecting and expanding clusters. The detection of clusters and pixel-type computation perform simultaneously. If sliding the threshold bar identifies a targeted pixel, the pixel becomes the first at the edge of a new cluster. Before defining (expanding) the cluster (i.e., discovering and identifying all connected pixels comprising an individual cluster), the app adds the putative cluster to a task pool that may contain additional clusters also awaiting definition (Blumofe et al. 1996). Every time a new cluster is added to the task pool, a new "work stealing" process initiates if not already in progress (Kleppner and Kolenkow 2013; Suksompong et al. 2016). This process expands the defined cluster by implementing another flood-fill algorithm (Treuenfels 1994). The work stealing algorithm

\begin{tabular}{|c|c|}
\hline Term & Definition \\
\hline Title & Title of the analysis assigned by the user \\
\hline Image name & Name of the image for analysis \\
\hline Disease ratio & $\begin{array}{l}\text { Percentage of image covered by the target } \\
\text { pixel colors of interest (i.e., percent } \\
\text { disease) }\end{array}$ \\
\hline Date & Date (and time) of the analysis \\
\hline Cluster exclusion criteria & $\begin{array}{l}\text { Relative size of clusters excluded from the } \\
\text { analysis. The user can specify this value } \\
\text { before performing the simulations. These } \\
\text { clusters represent anomalous or } \\
\text { meaningless groups of pixels within the } \\
\text { defined area. }\end{array}$ \\
\hline Number of clusters & $\begin{array}{l}\text { Number of clusters used to calculate } \\
\text { percent disease and to perform } \\
\text { simulations. The number is a function of } \\
\text { the value set for the cluster exclusion } \\
\text { criteria. }\end{array}$ \\
\hline Number of simulations & $\begin{array}{l}\text { Number of simulations specified by the } \\
\text { user for the analysis }\end{array}$ \\
\hline Mean inter-cluster distance & $\begin{array}{l}\text { Mean number of normalized units } \\
\text { separating clusters in the simulated } \\
\text { analysis }\end{array}$ \\
\hline $\begin{array}{l}\text { Mean simulated inter-cluster } \\
\text { distance }\end{array}$ & $\begin{array}{l}\text { Mean number of normalized units } \\
\text { separating clusters in the simulated } \\
\text { analysis }\end{array}$ \\
\hline$t$ test value & Value derived from the analysis \\
\hline Probability value & Value derived from the analysis \\
\hline Cluster number & $\begin{array}{l}\text { Each cluster is assigned a unique value; } 1 \\
\text { to } x\end{array}$ \\
\hline Angle & $\begin{array}{l}\text { Relative compass orientation of the long } \\
\text { axis of a cluster }\end{array}$ \\
\hline Pixel count & $\begin{array}{l}\text { Number of pixels within a cluster } \\
(\sim \text { cluster size })\end{array}$ \\
\hline Bounding frame & $\begin{array}{l}\text { Pixel dimensions of the length } \times \text { width } \\
\text { dimensions of a cluster }\end{array}$ \\
\hline Mass center & $\begin{array}{l}\text { Pixel point that defines the center of mass } \\
\text { for a cluster }\end{array}$ \\
\hline Long axis length & $\begin{array}{l}\text { Number of pixels in the long axis of a } \\
\text { cluster }\end{array}$ \\
\hline Short axis length & $\begin{array}{l}\text { Number of pixels in the short axis of a } \\
\text { cluster }\end{array}$ \\
\hline Cluster shape & Long to short axis ratio \\
\hline
\end{tabular}
assumes an environment where a computation schedules to a set of

TABLE 1. Definition of terms and variables used by the Cluster app for spatial analysis of pixelated data 
dedicated processors (Kleppner and Kolenkow 2013; Suksompong et al. 2016). In the Cluster app, the algorithm schedules computations onto a pool of tasks and worker threads assigned to the processors by an operating system scheduler. When pixel-type computation and cluster expansion processes terminate, clusters are stored in a separate array according to the order of identification. A flood-fill algorithm using four-way flooding defines the order within the queue (Treuenfels 1994).

Computing cluster attributes. The Cluster app calculates spatial attributes for each cluster and uses this information in simulations to determine whether an observed pattern of clusters is random or nonrandom. First, the location of each cluster relative to image origin and cluster frame is computed simultaneously during cluster definition (Fig. 2A and B). This enables calculation of the minimum and maximum position of the pixels for the cluster frame using the origin $\left(x_{\min }, y_{\min }\right)$ and size $\left(\right.$ width $=x_{\max }-x_{\min }$, length $\left.=y_{\max }-y_{\min }\right)$ of each cluster. The app calculates and specifies the center of mass of each cluster. The center of mass is the unique point where the weighted relative position of the distributed mass sums to zero (Kleppner and Kolenkow 2013). In the case of a pixel matrix $P_{i}$, $i=1, \ldots n$, each with mass $m_{i}=1$, located in space with coordinates $r_{i}, i=1, \ldots n$, the coordinates $R$ of the center of mass satisfies

$$
\sum_{i=1}^{n} m_{i}\left(r_{i}-R\right)=0
$$

and solving this equation for $R$ provides:

$$
R=\frac{1}{M} \sum_{i=1}^{n} m_{i} \cdot r_{i}
$$

where $m_{i}=1$ and $M$ is the sum of the masses of all pixels.

Cluster attributes. The identification of each cluster preserves the angle or orientation as the segment that connects the two most distal points positioned at the edges. This forms the longest axis within the cluster and may go beyond its edges, but begins and ends within the defined cluster (Fig. 2C). To compute the longest axis (cluster length), the cluster shape divides into multiple pieces of the same size in circular form around the center of mass $(n=2 \cdot L)$; where $L$ is the number of cut lines that split the shape. At the side margins of each cluster, the edge pixels parse by initially identifying the pixel furthest from the centroid (i.e., the center of mass). The computations accelerate within the CPU by parsing only the pixels at the edge of each cluster. When all extremal pixels compute for each cluster slice, the algorithm detects the greatest distance
A

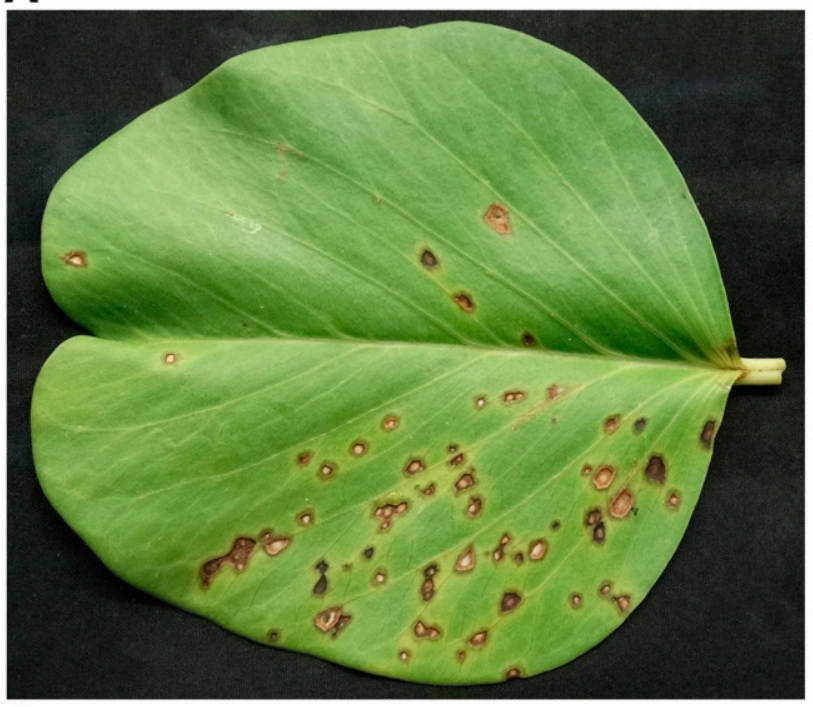

C

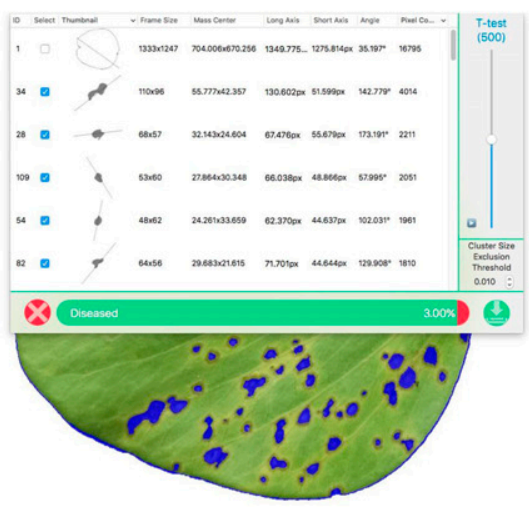

$\otimes \otimes \otimes$
B
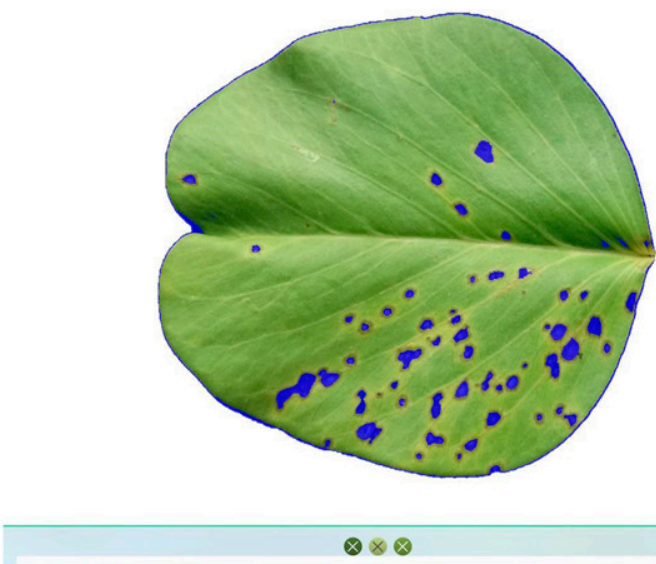

$\otimes \otimes \otimes$

D

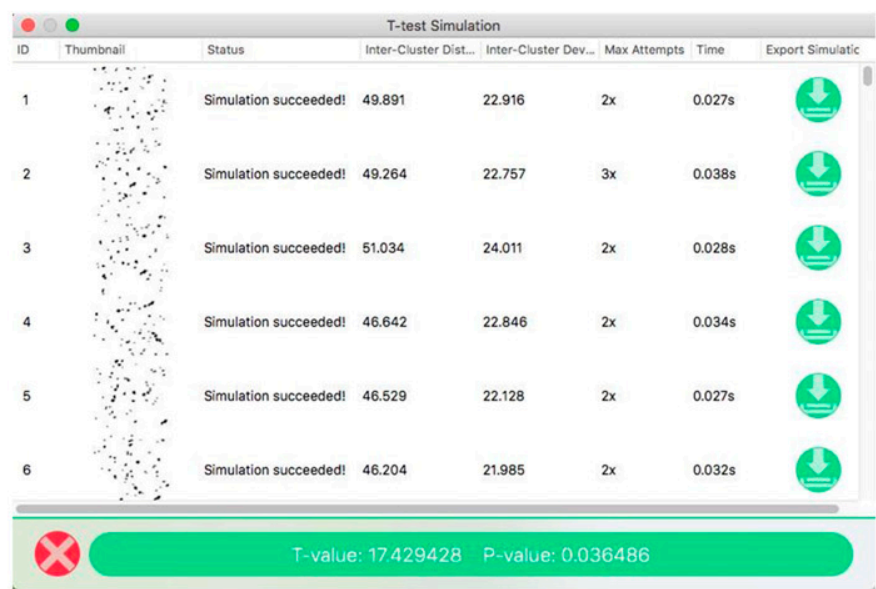

Fig. 3. Analysis of the spatial position of Cercospora leaf spot (CLS) lesions on a beach morning-glory leaf based on pixelated data using the Cluster app from A, a color photograph; B, use of the threshold slider to identify the target clusters (CLS lesions) and quantify disease severity. C, Output provided by the Cluster app is a summary of the selected target clusters and their attributes, and $\mathbf{D}$, results of the two-tailed $t$ test and associated probability including images of the spatial representation of the stochastic simulations. 
between the two points. By default, cluster shapes divide into 16 pieces, with $L=8$. The algorithm for finding the longest line is:

$$
\mathrm{O}(\mathrm{n})=N_{\text {edge }}+L \cdot(L-1)
$$

where $N_{\text {edge }}=$ number of edge pixels

The algorithm to calculate cluster width is similar to that described above for cluster length, except the calculated distance lies between the currently parsed pixel and the projection of that pixel on the cluster's longest axis. This infers the calculation will use only the furthest two pixels on the opposite sides of an individual cluster, referred to as the width axis' odd and even sides, respectively. Cluster width equals the distance between the odd side point and its projection on the length axis, summed with the distance between the even side point and its projection on the length axis (Fig. 2D), where $p$ is the detected extremal point and $p$ ' is its projection on the cluster's longest line:

$$
W_{\text {cluster }}=d\left(p_{\text {odd },} p_{\text {odd }}^{\prime}\right)+d\left(p_{\text {even },} p_{\text {even }}^{\prime}\right)
$$

Spatial analysis. The Cluster app uses cluster attribute data for analysis of spatial patterns following the user's specification of the cluster exclusion criterion. A cluster exclusion threshold of 0.01 infers that any target area less than $1 \%$ of the frame's pixel number represents anomalous clusters to be excluded from analysis. During simulations, the app places the centroid of each cluster successively at a random position within the frame in step-wise order of size, beginning with the largest cluster. The user specifies the number of simulations for the random placements of clusters to vary between 1 and 1,000. For each simulation, the app calculates the values for the mean and standard deviation of the inter-cluster distances between the centroids of the randomly placed clusters and the actual clusters in the image of interest. These data allow the calculation of a two-tailed $t$ test:

$$
t=\left(i c_{1}-i c_{2}-d\right) / s e
$$

where $i c=$ inter-cluster distance; $s e=$ standard error of the intercluster distance distribution; and $d=$ hypothesized difference between population means. The app also calculates an associated probability value with one degree of freedom $(d f)$ (Snedecor and Cochran 1991):

$$
P=\left[\frac{1}{(\sqrt{d f}) \mathrm{B}\left(\frac{1}{2}, d f / 2\right)}\right] \mathrm{lt}->-t \text { to } t \int\left(1+{\frac{x^{2}}{d f}}^{\left(-\frac{v+1}{2}\right)} . d x\right)
$$

The null hypothesis of the test is that defined clusters are distributed randomly within the defined frame. Rejection of the null hypothesis therefore provides statistical evidence of a nonrandom spatial pattern (Snedecor and Cochran 1991). The Cluster app produces cluster attributes and spatial analysis statistics as an output file in .csv format (Table 1).

Using the Cluster app for spatial pattern analysis. Example 1 - Photograph of a diseased leaf. The Cluster app analyzed the spatial pattern of Cercospora leaf spot (CLS) lesions on beach morning-glory (Ipomoea pes-caprae subsp. brasiliensis) depicted in a digital photograph. Beach morning-glory is a creeping vine commonly found just above the high-water mark on sandy Hawaiian beaches (Neal 1991). The incidence and severity of CLS caused by $C$. ipomoea (R. Shivas, personal communication) vary according to the suitability of conditions for infection and disease spread (Nelson 2013; Stevens 1925). We used the Cluster app to test the null hypothesis that clusters (i.e., lesions) were distributed randomly within the frame of interest defined by the beach morning-glory leaf (Fig. 3A). After identification of target clusters (i.e., disease lesions) (Fig. 3B), the example spatial analysis applied
500 stochastic simulations and a cluster size exclusion threshold of 0.01 (Fig. 3C).

Example 2 - Aerial photograph of a diseased crop field. Tan spot is a foliar disease that causes defoliation in lima bean (Phaseolus lunatus) fields in New York. The disease is caused by two pycnidial fungi: Boeremia exigua var. exigua (Gorny et al. 2015) and Didymella americana (Gorny et al. 2016). An aerial photograph of a lima bean field affected by tan spot was taken with a Canon EOS 6D camera with a multispectral sensor with three bands (green [470 to $570 \mathrm{~nm}$ ], blue [370 to $550 \mathrm{~nm}$ ], and red edge [660 to $770 \mathrm{~nm}$ ]) using a 50-mm lens (focal length $=24 \mathrm{~mm}$ ) from a fixed-wing aircraft flying at an elevation of $457 \mathrm{~m}$ (Fig. 4). The field location was near Pavilion, New York. The original photograph was $5,472 \times 3,648$ pixels, with an $855.62 \mathrm{~mm}^{2}$ radiometric resolution and reprocessed to $60.9 \mathrm{~mm}$ per pixel. Data were rescaled following atmospheric correction and radiometric calibration methods from 1 to 255 (8-bit data) for visualization of canopy reflectance using the normalized difference vegetative index [NDVI $\left.=\left(R_{\text {red }}-R_{\text {blue }}\right) /\left(R_{\text {red }}+R_{\text {blue }}\right)\right]$ (Asrar et al. 1984; Myneni et al. 1995; Sellers 1985).

We analyzed the spatial pattern of tan spot foci (individual and groups of diseased plants) within the field by using the Cluster app at 500 stochastic simulations and repeated sequentially for comparison using cluster size exclusion thresholds of 0.01 and 0.001 , respectively. The null hypothesis for each analysis was that tan spot foci were randomly distributed $(P>0.05)$ within the field.

Example 3 - Landscape features using satellite imagery. To explore the utility of using spatial analysis of pixelated data within the Cluster app for ecological applications using satellite imagery, we obtained from Google Maps (https://maps.google.com/maps; Fig. 5A) a rectangular photographic section of landscape depicting the distribution of lakes in Minnesota. The clusters (lakes) were identified by color designation of the nontarget areas and the use of the threshold slider (Fig. 5B). The null hypothesis of this analysis was that lakes were distributed randomly $(P>0.05)$ within the frame. The analysis was conducted with a cluster exclusion threshold of 0.01 and 500 stochastic simulations. The app also summarized data

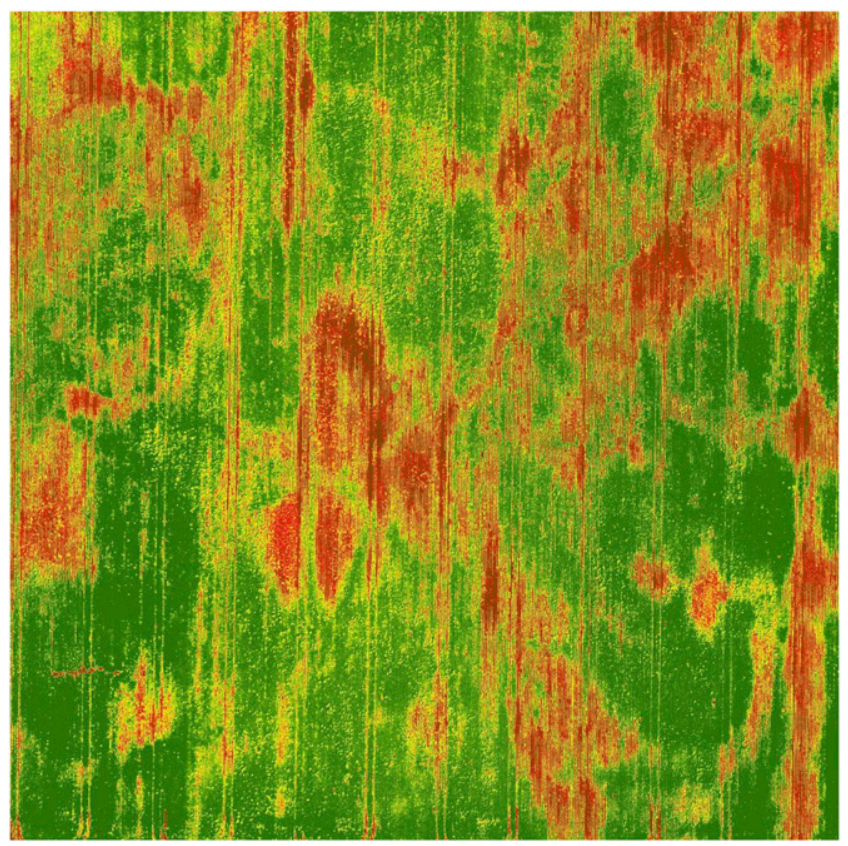

Fig. 4. Image of a lima bean field affected by tan spot in New York taken from a fixed-wing aircraft at $457 \mathrm{~m}$ using a Canon EOS 6D camera with a multispectral sensor. The image was converted to canopy reflectance using the normalized difference vegetative index. Dark pixels represent areas with less photosynthetic capacity as a result of defoliation of lima bean plants affected by tan spot. 
regarding the various attributes of the clusters (size, shape, number, orientation, etc.).

Example 4 - Ecological analysis using satellite imagery. We applied the Cluster app to analyze the spatial distribution of an infestation of perennial weeds in an abandoned pineapple field on the island of Maui, Hawai'i. A satellite image of the field from Google Maps (https://maps.google.com/maps) subjected to Adobe Creative Cloud Photoshop CC (Adobe Systems Incorporated, San Jose, CA) helped to select the portion of the field of interest, sharpen the image, and correct for exposure and inconsistencies in hue. The abandoned field had been infested by a species of weedy trees, indicated by the dark green color (Fig. 6A). Using a cluster exclusion threshold of 0.001 and 500 simulations, we tested the null hypothesis of a random spatial pattern of clusters.

\section{RESULTS}

Example 1 - Photograph of a diseased leaf. The symptomatic area of the beach morning-glory leaf with CLS was 3\% (Fig. $3 \mathrm{~A}$ to $\mathrm{C}$ ). Spatial analysis resulted in an estimated $t$ value of 17.4 $(P=0.04$; Fig. 3D). This result rejected the null hypothesis of a random cluster distribution within the image. Therefore, we defined the spatial pattern of CLS lesions on the beach morning-glory leaf as nonrandom.

Example 2 - Aerial photograph of a diseased field. Adjustment of the cluster exclusion threshold between 0.01 and 0.001 prior to spatial analysis of tan spot foci in the aerial image of the lima bean field changed percent disease and the number of target areas included in the analysis. When the cluster exclusion threshold was 0.01 , the percent disease in the image was $10.61 \%$ and the number of included and excluded clusters was 90 and 1,036 , respectively. Changing the cluster exclusion threshold to 0.001 increased percent disease to $12.02 \%$ and the number of included and excluded clusters was 588 and 538, respectively. Therefore, changing the cluster exclusion threshold from 0.01 to 0.001 added 498 clusters. In both cases, the probability values were greater than 0.05 ( $P=0.6$ at a threshold of $0.01 ; P=0.2$ at a threshold of 0.001$)$, suggesting a random spatial arrangement of clusters within the field.

Example 3 - Landscape features using satellite imagery. The original satellite image (Fig. 5A) interrogated with the threshold slider function (Fig. 5B) identified $7.21 \%$ of the area within the image covered by lakes (Fig. 5C). The analysis of the spatial arrangement of lakes in the image using a cluster exclusion threshold of 0.01 with 500 simulations (Fig. 5C) produced a probability value exceeding 0.05
A

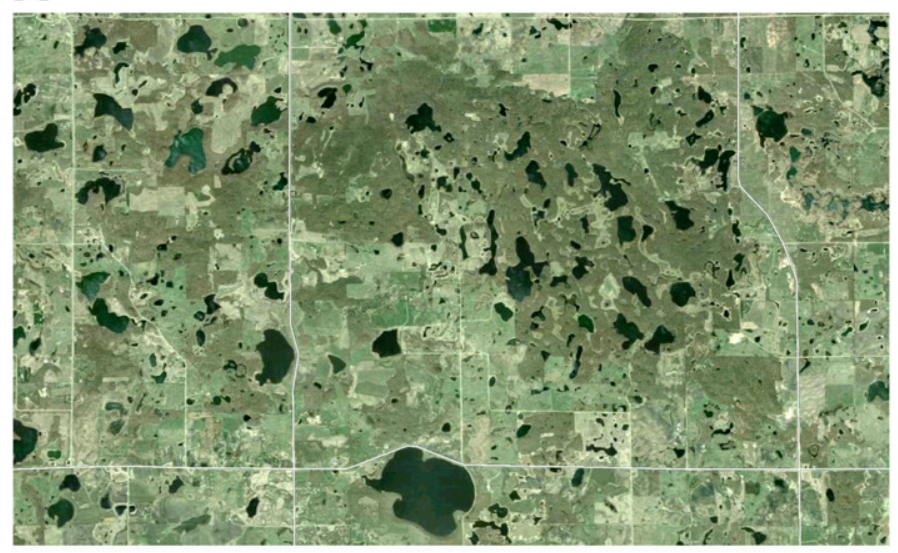

C

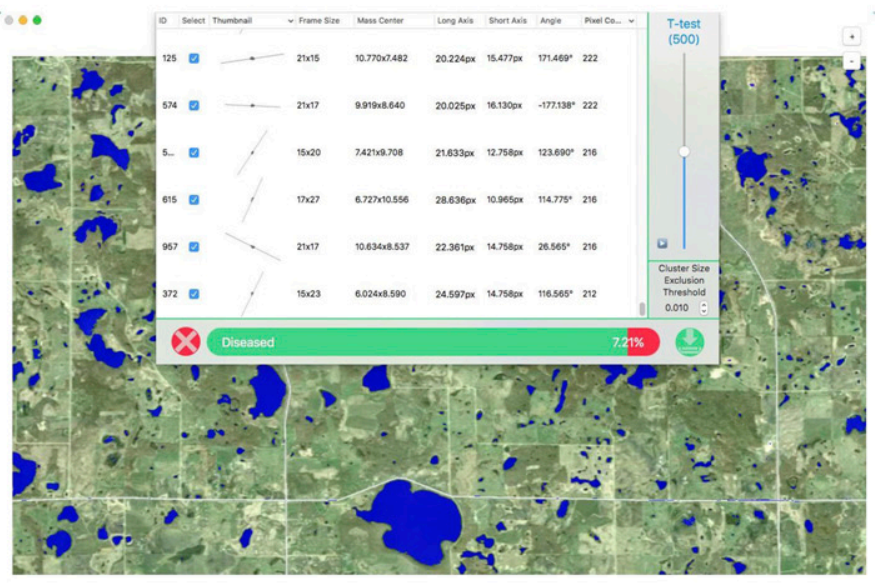

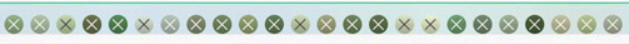

B

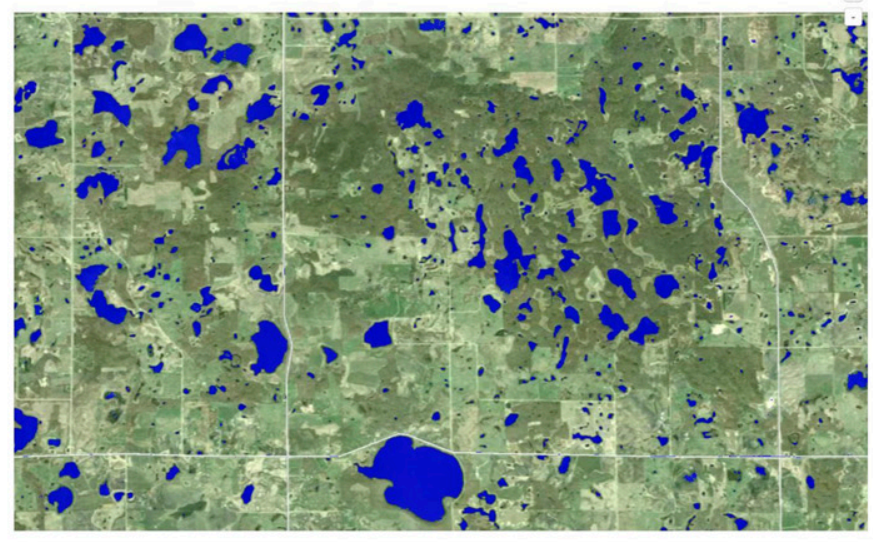

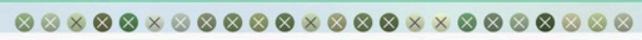

D

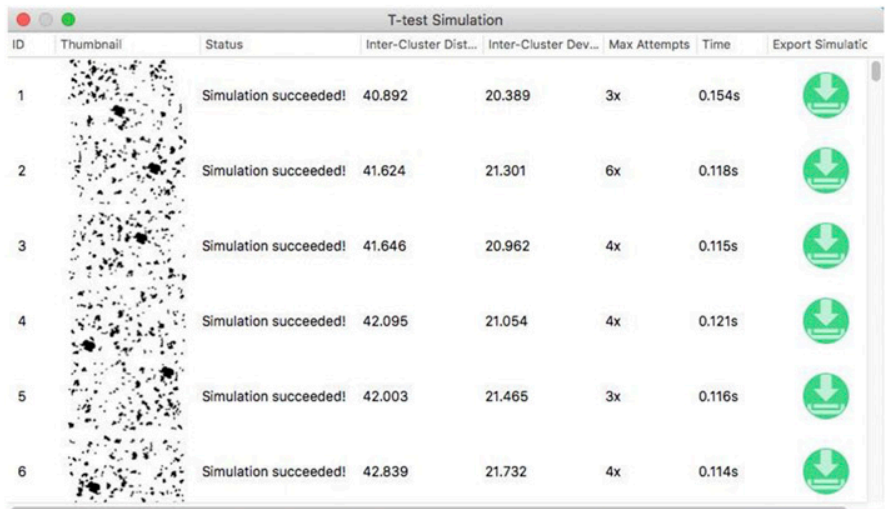

8

T-value: 1.593908 P-value: 0.356708

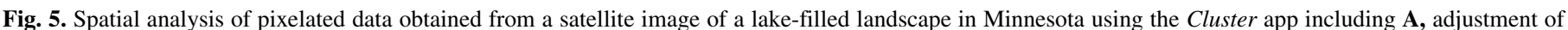

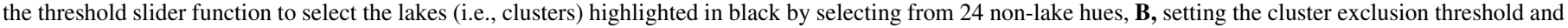

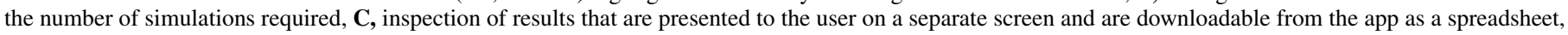

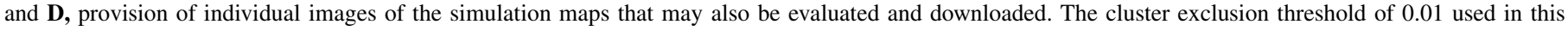
analysis means any target cluster (lake) occupying less than $1 \%$ of the frame area was excluded from the analysis. 
$(P=0.36$; Fig. 5D). Therefore, the null hypothesis was accepted and the arrangement of the lakes in the image concluded to be random. Details of the cluster attributes provided by the Cluster app are shown in Table 2.

Example 4 - Ecological analysis using satellite imagery. The app analyzed the spatial pattern of the distribution of weedy trees in the satellite image of the abandoned pineapple field (Fig. 6A) following definition of the target clusters (weeds) using the threshold slider function of the Cluster app (Fig. 6B). Spatial analysis produced a probability value slightly greater than 0.05 $(P=0.058)$, suggesting the null hypothesis should be accepted and therefore conclusion that the weedy tree species in the image were distributed randomly (Fig. 6C). An example of the output also provided by the Cluster app as a downloadable .csv file is shown in Table 3 .

\section{DISCUSSION}

To our knowledge, direct interrogation of the spatial patterns of epiphytotics using pixelated images as embodied in Cluster is a unique and novel analytical method having potentially broad application to the biological sciences (Table 4). We included the approach in a desktop computer application to facilitate ease of use through interactive functions. This discounts the need for any user knowledge of coding needed for implementing comparable approaches utilizing open access image analysis and statistical platforms. The $t$ test used in Cluster is fundamentally similar to other methods used in plant pathology or ecology that analyze binary or quadrat-based data (Collett 2003; Madden et al. 2007; Pielou 1977; Ripley 1981). These approaches conduct spatial analyses based on the presence or absence of a species, or a quality or quantity within a superimposed quadrat in a field or habitat. In the Cluster app, the quadrats are the individual pixels having natural, targeted colors. These pixelated quadrats differ from the arbitrarily defined areas in subsections of a field or landscape. The Cluster approach facilitates the analysis of areas with enhanced spatial resolution. The app has the power to rapidly define up to 24 colors or

TABLE 2. Cluster attribute data provided by the Cluster app upon analysis of the spatial arrangement of lakes in Minnesota from a satellite image

\begin{tabular}{lc}
\hline Cluster attribute & Value $^{\mathrm{a}}$ \\
\hline Mean inter-lake distance & 40.25 units \\
Mean simulated inter-lake distance & 41.77 units \\
Mean simulated inter-lake distance & 21.20 units \\
$\quad$ deviation & 119 \\
Number of lakes analyzed for pattern & 1,060 \\
Number of anomalous clusters excluded & $1,270.2(212$ to 24,403$)$ \\
Mean pixel count (i.e., relative lake size) & $90.04^{\circ}\left(-177.14^{\circ}\right.$ to $\left.171.47^{\circ}\right)$ \\
Compass orientation of the long axis & $1.74(1.05$ to 5$)$ \\
Lake shape (long/short axis ratio) &
\end{tabular}

a Range provided in parentheses.
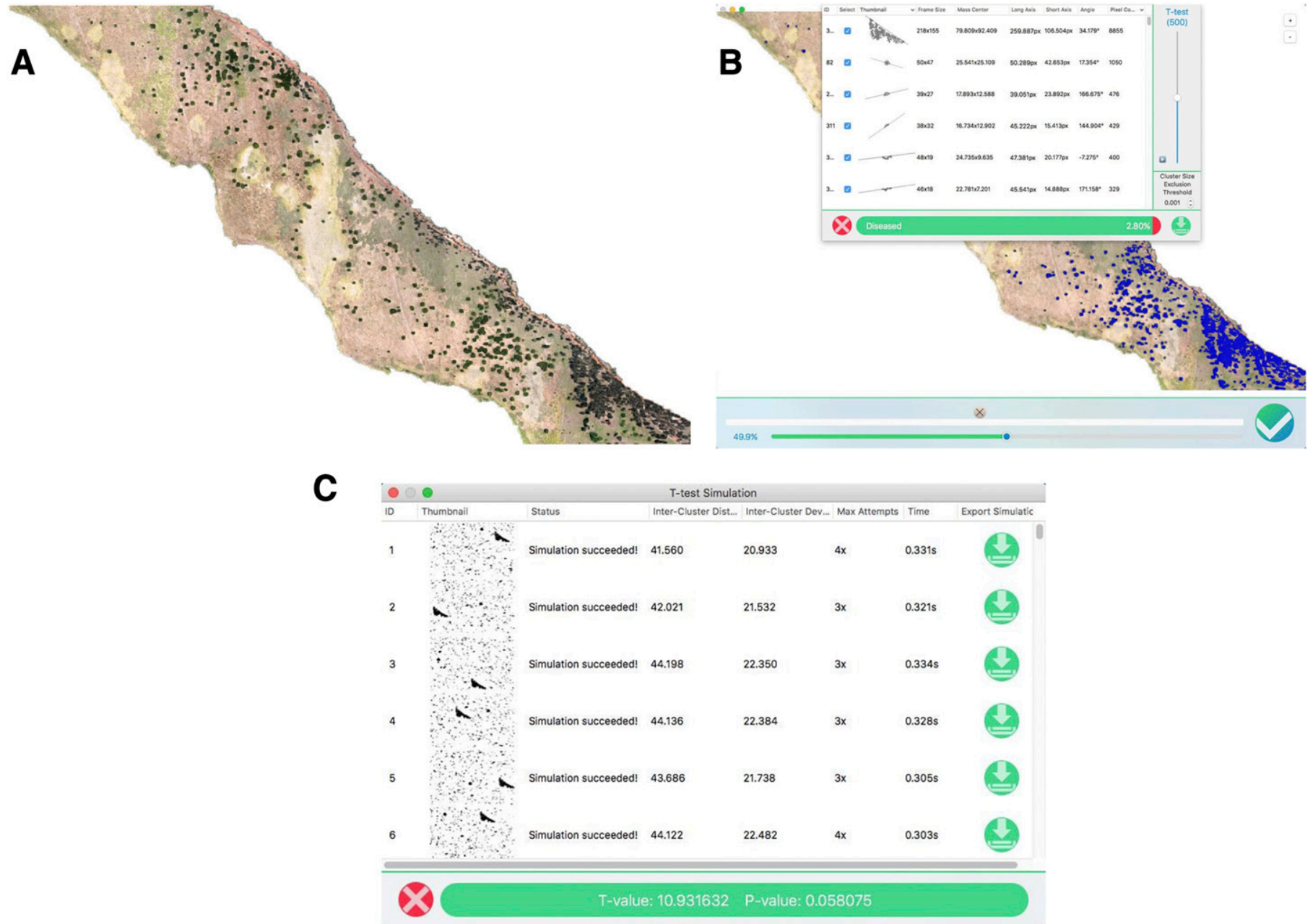

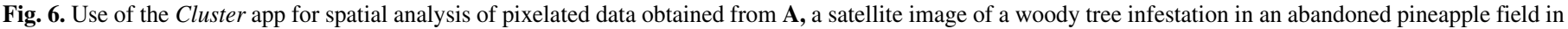

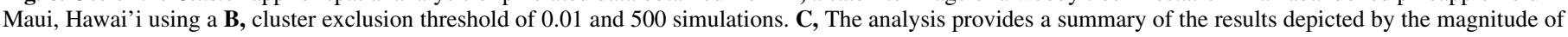

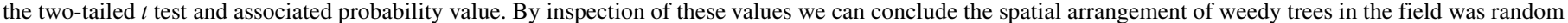
$(P>0.05)$. 
hues for pixels. This can reduce the target and nontarget pixels in a complex photograph to binary data for pattern analysis, which differs from traditional quadrat-based pattern approaches. However, by applying Cluster, a potential trade-off is that the perceived colors of an object may be affected by incident or reflected light, shade, debris, soils, nontarget pests, plant genetics or other qualities. As a result, users may further manipulate an image using imaging software. However, the ability to select multiple colors within the Cluster app minimizes the detriments of this potential complication.

The utility of the approach implemented within the Cluster app became apparent in the presentation of several plant pathology scenarios that spanned varying spatial resolutions, from digital photographs of a single leaf to satellite imagery of an entire field. This technique may have the greatest potential in quantifying epidemics or spatial patterns in challenging landscapes or scales. Obtaining sufficient resolution for spatial analysis is particularly problematic in these situations where it is difficult to establish transects or plots such as forestry plantations, natural ecosystems, and across entire fields in broad-acre cropping systems.

In example 1, the potential of this approach to interrogate the attributes of CLS lesions and overall spatial patterns at the single leaf scale was demonstrated, similar to the approach used by Schwanck and Del Ponte (2016). By the provision of cluster attribute statistics (size, shape, and number), the Cluster app enables exploration of

TABLE 3. Summary statistics produced by the Cluster app following spatial analysis of a weedy tree infestation of a pineapple field in Maui, Hawai'i

\begin{tabular}{lc}
\hline Output summary statistics & Value \\
\hline Diseased ratio & $2.8 \%$ \\
Cluster exclusion criteria & $<0.001$ \\
Number of clusters included & 335 \\
Number of clusters excluded & 136 \\
Number of simulations & 500 \\
Mean inter-cluster distance & 32.655 units \\
Mean simulated inter-cluster distance & 43.31 units \\
Mean simulated inter-cluster distance & 21.789 units \\
deviation & \\
$t$ test value & 10.93 \\
Probability value & 0.058 \\
\hline
\end{tabular}

epidemiological hypotheses such as the coalescence of individual lesions over time and temporal changes in disease intensity and among environments. The approach could also be used to explore a broader spatiotemporal understanding of disease progress in a spatial hierarchy within a plant population or on different leaves within the canopy.

Example 2 demonstrated the potential of spatial analysis of pixelated data for an epidemic of tan spot of lima bean on a field scale. This approach could be used to facilitate addressing questions concerning the dominant inoculum sources and impact of cluster size on crop loss on a field scale. This information is essential for the development of evidence-based and location-specific disease management strategies and tactics (Hughes 1987, 2017). Similarly, spatial data for geographical features within a landscape such as that illustrated for the geospatial arrangement of lakes in Minnesota (example 3) may facilitate ecological research questions such as geological and/or ecological factors contributing to compass orientation of the long axes of the targets.

The interpretations and inferences from a spatial pattern analysis are strongly dependent on scale and hierarchical level used for analysis (Turechek and McRoberts 2013). For example, the spatial patterns observed within an arbitrarily selected frame size will have an equally pointless meaning that may lead to bias and false conclusions. Users are therefore urged to consider the effect of frameshift on the biological conclusions when using this approach and carefully assess and define the most appropriate frame size and dimensions for the biological, ecological or physiographical meaning to derive relevant conclusions about the system of interest (Table 4).

A limitation of this approach is for data obtained from sampling units arranged in a grid matrix, such as the row cropping system of the lima bean field (example 2). In this scenario, the target units (i.e., plants) were not distributed continuously in the frame and instead were restricted to predetermined rows. During the random simulations, symptomatic target plants have an equal probability of being assigned to any location within the frame, including placement to areas between rows, which may lead to biased conclusions about spatial patterns. This effect may be magnified in lowaltitude aerial photographs because of higher spatial resolution. For example, wider spacing between rows at the higher resolution would allow for more space in the image devoid of plants.

TABLE 4. Research areas in plant disease epidemiology with hypotheses testable using the Cluster app

\begin{tabular}{|c|c|c|}
\hline Research area & Description & Example null hypothesis $\left(H_{o}\right)$ \\
\hline Comparative epidemiology & $\begin{array}{l}\text { Comparisons among and between } \\
\text { pathosystems and environments }\end{array}$ & $\begin{array}{l}\text { Patterns vary among pathosystems } \\
\text { Patterns vary among environments }\end{array}$ \\
\hline Temporal analysis & $\begin{array}{l}\text { Quantification of spatial attributes of an } \\
\text { epidemic }\end{array}$ & $\begin{array}{l}\text { Cluster size changes over time } \\
\text { Cluster shape changes over time }\end{array}$ \\
\hline Spatiotemporal patterns & $\begin{array}{l}\text { Quantify changes in patterns and cluster } \\
\text { attributes over time }\end{array}$ & $\begin{array}{l}\text { Clusters enlarge over time } \\
\text { Patterns change over time from random } \\
\text { to aggregated }\end{array}$ \\
\hline Treatment effects & $\begin{array}{l}\text { Examination of the effects of treatments } \\
\text { on spatial pattern }\end{array}$ & $\begin{array}{l}\text { Use of a pesticide changes the cluster } \\
\text { attributes and spatial patterns of a disease }\end{array}$ \\
\hline Spatial scale & $\begin{array}{l}\text { Analysis of spatial patterns at different } \\
\text { scales of resolution }\end{array}$ & $\begin{array}{l}\text { Changes in pattern at the leaf scale } \\
\text { correspond to changes in pattern at the } \\
\text { plant population scale }\end{array}$ \\
\hline Strength of nonrandomness & Quantify the strength of nonrandomness & $\begin{array}{l}\text { The } P \text { value is a measure of the strength } \\
\text { of nonrandomness }\end{array}$ \\
\hline Cluster shape and orientation & Quantify cluster shape and orientation & $\begin{array}{l}\text { The shape of clusters for disease } x \text { differ } \\
\text { from disease } y\end{array}$ \\
\hline Interspecific patterns & $\begin{array}{l}\text { Quantify the patterns of differing targets } \\
\text { in a frame }\end{array}$ & Patterns differ for distinct targets in a frame \\
\hline
\end{tabular}


Moreover, since plants are restricted to rows, the long axes of clusters have a higher probability of coinciding with row directions. The potential trade-off here is the enhanced ability of this approach to evaluate larger areas of interest and improve the resolution of nontarget areas based on hue and color.

Example 4 demonstrated the utility of Cluster to analyze spatial patterns within a continuous frame. In contrast to the row cropping system (example 3), the targets (i.e., weeds) had an equal probability of occurring at any point within the frame with a null hypothesis of a random spatial pattern. This approach offers benefits over traditional transect-based methods currently used in ecology and weed science to quantify local-scale spatial patterns of pest incursions or landscape diversity based on the placement of arbitrary transects and the time required for data collection over large and diverse areas (Greig-Smith 1952; Lichstein et al. 2002; Ripley 1981). Multiple targets (different weed species) could be analyzed independently within a frame to generate comparative hypotheses related to interspecific relations.

Users should consider carefully the appropriate frame, size and resolution for biological relevance within the system of interest and examination of the sensitivity of variables that may influence conclusions (Table 4). These variables include the cluster exclusion threshold, number of random simulations, and appropriateness of the $5 \%$ probability level for identifying evidence for rejecting the null hypothesis in support of a nonrandom spatial pattern (Madden et al. 2015).

The Cluster app is available for free download from the iTunes store at https://sites.google.com/a/hawaii.edu/cluster/. The minimum macOS version to run the application is macOS 10.9 (Mac OS $\mathrm{X}$ Mavericks). The hardware requirements for the support of Mac OS X Mavericks are $2 \mathrm{~GB}$ of memory and $8 \mathrm{~GB}$ of available space. Supported device models include iMac (at least mid-2007); MacBook (at least 2008 Aluminum, or early 2009); MacBook Pro (at least mid/ late 2007); Xserve (early 2009); MacBook Air (at least late 2008); Mac mini (at least early 2009); and Mac Pro (at least early 2008). A device with a minimum of a dual-core i5 processor, $8 \mathrm{~GB}$ of memory and a dedicated graphics card is recommended.

\section{ACKNOWLEDGMENTS}

Funding for development of the Cluster app was provided by the United States Department of Agriculture, National Institute of Food and Agriculture (USDA-NIFA) Hatch project NYG-625424, managed by the New York State Agricultural Experiment Station, Cornell University, Geneva, NY, and USDA-NIFA Hatch project HAW08036-H (Accession no. 1010616) administered by the College of Tropical Agriculture and Human Resources, University of Hawai 'i at Mānoa. We thank D. Ionel (Adelante Consulting Inc., Canada) for assistance with app coding and design.

\section{LITERATURE CITED}

Asrar, G. E., Fuchs, M., Kanemasu, E. T., and Hatfield, J. L. 1984. Estimating absorbed photosynthetic radiation and leaf area index from spectral reflectance in wheat. Agron. J. 76:300-306.

Baddeley, A., and Turner, R. 2005. Spatstat: An R package for analyzing spatial point patterns. J. Stat. Softw. 12:1-42.

Barbedo, J. G. A. 2013. Digital image processing techniques for detecting, quantifying and classifying plant diseases. Springerplus 2:660.

Barbedo, J. G. A. 2014. An automatic method to detect and measure leaf disease symptoms using digital image processing. Plant Dis. 98: 1709-1716.

Blumofe, R. D., Joerg, C. F., Kuszmaul, B. C., Leiserson, C. E., Randall, K. H., and Zhou, Y. 1996. Cilk: An efficient multithreaded runtime system. J. Par. Distr. Comp. 37:55-69.

Bock, C. H., Poole, G. H., Parker, P. E., and Gottwald, T. R. 2010. Plant disease severity estimated visually, by digital photography and image analysis, and by hyperspectral imaging. Crit. Rev. Plant Sci. 29:59-107.

Collett, D. 2003. Modelling Binary Data, 2nd Ed. CRC Press, Boca Raton, FL.

Ferrandino, F. J. 1996. Two-dimensional distance class analysis of diseaseincidence data: Problems and possible solutions. Phytopathology 86:685-691.
Ferrandino, F. J. 1998. Past nonrandomness and aggregation to spatial correlation: 2DCORR, a new approach for discrete data. Phytopathology 88: 84-91.

Garg, R. P., and Sharapov, I. 2002. Techniques for Optimizing Applications High Performance Computing. Sun Blueprints. Prentice-Hall PTR, NJ.

Gibbons, J., and Chakraborti, S. 2010. Nonparametric Statistical Inference. Chapman and Hall/CRC Press, Boca Raton, FL.

Gorny, A. M., Kikkert, J. R., Dunn, A. R., Dillard, H. R., Smart, C. D., and Pethybridge, S. J. 2015. Tan spot of lima bean caused by Boeremia exigua in New York State. Can. J. Plant Pathol. 37:523-528.

Gorny, A. M., Kikkert, J. R., Shivas, R. G., and Pethybridge, S. J. 2016. First report of Didymella americana on lima bean (Phaseolus lunatus). Can. J. Plant Pathol. 38:389-394.

Gosme, M., and Lucas, P. 2009a. Cascade: An epidemiological model to simulate disease spread and aggregation across multiple scales in a spatial hierarchy. Phytopathology 99:823-832.

Gosme, M., and Lucas, P. 2009b. Disease spread across multiple scales in a spatial hierarchy: Effect of host spatial structure and of inoculum quantity and distribution. Phytopathology 99:833-839.

Gottwald, T. R., Richie, S. M., and Campbell, C. L. 1992. LCOR2-Spatial correlation analysis software for the personal computer. Plant Dis. 76: 213-215.

Gower, J. C. 1982. Euclidean distance geometry. Math. Sci. 1:1-14.

Gower, J. C. 1985. Properties of Euclidean and non-Euclidean distance matrices. Lin. Algeb. Appl. 67:81-97.

Gray, S. M., Moyer, J. W., and Bloomfield, P. 1986. Two-dimensional distance class model for quantitative description of virus-infected plant distribution lattices. Phytopathology 76:243-248.

Greig-Smith, P. 1952. The use of random and contiguous quadrats in the study of the structure of plant communities. Ann. Bot. (Lond.) 16:293-316.

Hughes, G. 1987. Spatial heterogeneity in crop loss assessment models. Phytopathology 78:883-884.

Hughes, G. 2017. The evidential basis of decision making in plant disease management. Annu. Rev. Phytopathol. 55:2.1-2.19.

Hughes, G., and Madden, L. V. 1992. Aggregation and incidence of disease. Plant Pathol. 41:657-660.

Hughes, G., and Madden, L. V. 1993. Using the beta-binomial distribution to describe aggregated patterns of disease incidence. Phytopathology 83: 759-763.

Hughes, G., McRoberts, N., Madden, L. V., and Gottwald, T. R. 1997. Relationships between disease incidence at two levels in a spatial hierarchy. Phytopathology 87:542-550.

Hunter, R. S. 1948a. Photoelectric color difference meter. J. Opt. Soc. Am. 38:661.

Hunter, R. S. 1948b. Accuracy, precision and stability of new photo-electric color-difference meter. J. Opt. Soc. Am. 38:1094.

Isaaks, E. H., and Srivastava, R. M. 1989. Applied Geostatistics. Oxford University Press, New York.

Jain, A. K. 1989. Fundamentals of Image Processing. Prentice Hall, NJ.

Kleppner, D., and Kolenkow, R. 2013. An Introduction to Mechanics, 2nd Ed. Cambridge University Press, UK.

Kriss, A. B., Paul, P. A., and Madden, L. V. 2012. Characterizing heterogeneity of disease incidence in a spatial hierarchy: A case study from a decade of observations of Fusarium head blight of wheat. Phytopathology 102: 867-877.

Kuska, M., Wahabzada, M., Leucker, M., Dehne, H.-W., Kersting, K., Oerke, E.-C., Steiner, U., and Mahlein, A.-K. 2015. Hyperspectral phenotyping on the microscopic scale: Towards automated characterization of plant-pathogen interactions. Plant Methods 11:28.

Lamari, L. 2002. Assess: Image Analysis Software for Plant Disease Quantification. American Phytopathological Society, St. Paul, MN.

Leucker, M., Mahlein, A.-K., Steiner, U., and Oerke, E.-C. 2016. Improvement of lesion phenotyping in Cercospora beticola-sugar beet interaction by hyperspectral imaging. Phytopathology 106:177-184.

Li, B., Madden, L. V., and Xu, X. 2012. Spatial analysis by distance indices: An alternative local clustering index for studying spatial patterns. Methods Ecol. Evol. 3:368-377.

Lichstein, J. W., Simons, T. R., Shriner, S. A., and Franzreb, K. E. 2002. Spatial autocorrelation and autoregressive models in ecology. Ecol. Monogr. 72:445-463.

Madden, L. V., and Hughes, G. 1994. BBD-computer software for fitting the beta-binomial distribution to disease incidence data. Plant Dis. 78:536-540.

Madden, L. V., and Hughes, G. 1995. Plant disease incidence: Distributions, heterogeneity, and temporal analysis. Annu. Rev. Phytopathol. 33:529-564.

Madden, L. V., and Hughes, G. 1999. An effective sample size for predicting plant disease incidence in a spatial hierarchy. Phytopathology 89:770-781.

Madden, L. V., Hughes, G., and van den Bosch, F. 2007. The Study of Plant Disease Epidemics. American Phytopathological Society, St. Paul, MN.

Madden, L. V., Louie, R., Abt, J. J., and Knoke, J. K. 1982. Evaluation of tests for randomness of infected plants. Phytopathology 72:195-198. 
Madden, L. V., Shah, D. A., and Esker, P. D. 2015. Does the $P$ value have a future in plant pathology? Phytopathology 105:1400-1407.

Mahlein, A.-K., Steiner, U., Dehne, H.-W., and Oerke, E.-C. 2010. Spectral signatures of sugar beet leaves for the detection and differentiation of diseases. Precis. Agric. 11:413-431.

Mahlein, A.-K., Steiner, U., Hillnhuetter, C., Dehne, H.-W., and Oerke, E.-C. 2012. Hyperspectral imaging for small-scale analysis of symptoms caused by different sugar beet diseases. Plant Methods 8:3.

McRoberts, N., Hughes, G., and Savary, S. 2003. Integrated approaches to understanding and control of diseases and pests in field crops. Austral. Plant Pathol. 32:167-180.

Morisita, M. 1959. Measuring of the dispersion of individuals and the distribution patterns. Mem. Fac. Sci. Kyushu Univ. Ser. E 2:215-235.

Mutka, A. M., and Bart, R. S. 2015. Image-based phenotyping of plant disease symptoms. Front. Plant Sci. 5:734

Myneni, R. B., Hall, F. B., Sellers, P. J., and Marshak, A. L. 1995. The interpretation of spectral vegetation indices. IEEE Trans. Geosci. Remote Sens. 33:481-486.

Neal, M. C. 1991. In gardens of Hawaii. Bernice P. Bishop Museum, Special Publication 50. Bishop Museum Press, Honolulu, HI.

Nelson, S. C. 1996. A simple analysis of disease foci. Phytopathology 86: 332-339.

Nelson, S. C. 2013. Leaf spot of beach morning-glory. University of Hawaii at Manoa, College of Tropical Agriculture and Human Resources, Cooperative Extension Service PD-95.

Nelson, S. C., Marsh, P. L., and Campbell, C. L. 1992. 2DCLASS, a twodimensional distance class analysis software for the personal computer. Plant Dis. 76:427-432.

O'Rourke, J. 1985. Finding minimal enclosing boxes. Int. J. Comp. Inf. Sci. 14:183-199.

Perry, J. N. 1995. Spatial analysis by distance indices. J. Anim. Ecol. 64: 303-314.

Perry, J. N. 1998. Measures of spatial pattern for counts. Ecology 79:1008-1017.

Perry, J. N., Winder, L., Holland, J. M., and Alston, R. D. 1999. Red-blue plots for detecting clusters in count data. Ecol. Lett. 2:106-113.

Pethybridge, S. J., and Nelson, S. C. 2015. Leaf Doctor: A new portable application for quantifying plant disease severity. Plant Dis. 99: 1310-1316.

Pielou, E. C. 1977. Mathematical Ecology. John Wiley \& Sons, New York.

Ripley, B. D. 1981. Modeling spatial patterns. J. Roy. Stat. Soc. B 39:172-212.

Schwanck, A. A., and Del Ponte, E. M. 2016. Measuring lesion attributes and analyzing their spatial patterns and the leaf scale using digital image analysis. Plant Pathol. 65:1498-1508.
Sellers, P. J. 1985. Canopy reflectance, photosynthesis, and transpiration. Int. J. Remote Sens. 6:1335-1372.

Sezgin, M., and Sankur, B. 2004. Survey over image thresholding techniques and quantitative performance evaluation. J. Electr. Imag. 131:146-165.

Shah, D. A., Dillard, H. R., and Pethybridge, S. J. 2017a. Probability distributions for marketable pods and white mould on snap bean. Ann. Appl. Biol. 171:179-189.

Shah, D. A., Dillard, H. R., and Pethybridge, S. J. 2017b. Hierarchical models for white mould in snap bean. Plant Pathol. doi:10.1111/ppa.12724

Sharma, G. 2003. Digital Color Imaging Handbook. CRC Press, Boca Raton, FL.

Snedecor, G. W., and Cochran, W. G. 1991. Statistical Methods, 8th Ed. Wiley-Blackwell Publishing, New York.

Stevens, F. L. 1925. Hawaiian Fungi. Bernice P. Bishop Museum Bulletin 19: 1-189. Bishop Museum Press, Honolulu, HI.

Suksompong, W., Leiserson, C. E., and Schardl, T. B. 2016. On the efficiency of localized work stealing. Inf. Process. Lett. 116:100-106.

Swed, F., and Eisenhart, C. 1943. Tables for testing randomness of grouping in a sequence of alternatives. Ann. Math. Stat. 14:66-87.

Treuenfels, A. 1994. An efficient flood visit algorithm. C/C++ Users J. 12: 39-66.

Turechek, W. W., and Madden, L. V. 1999a. Spatial pattern analysis of strawberry leaf blight in perennial production systems. Phytopathology 89: 421-433.

Turechek, W. W., and Madden, L. V. 1999b. Spatial pattern analysis and sequential sampling for the incidence of leaf spot on strawberry in Ohio. Plant Dis. 83:992-1000

Turechek, W. W., and Madden, L. V. 2003. A generalized linear modeling approach for characterizing disease incidence in a spatial hierarchy. Phytopathology 93:458-466.

Turechek, W. W., Madden, L. V., Gent, D. H., and Xu, X.-M. 2011. Comments regarding the binary power law for heterogeneity of disease incidence. Phytopathology 101:1396-1407.

Turechek, W. W., and Mahaffee, W. 2004. Spatial pattern analysis of hop powdery mildew in the Pacific Northwest: Implications for sampling. Phytopathology 94:1116-1128.

Turechek, W. W., and McRoberts, N. 2013. Considerations of scale in the analysis of spatial pattern of plant disease epidemics. Annu. Rev. Phytopathol. 51:453-472.

Willocquet, L., and Savary, S. 2004. An epidemiological simulation model with three scales of spatial hierarchy. Phytopathology 94:883-891.

Wolfert, S., Ge, L., Verdouw, C., and Bogaardt, M.-J. 2017. Big data in smart farming-A review. Agric. Syst. 153:69-80. 